\title{
Urgences
}

\section{Texte à deux voix : l'enseignement de la littérature, lire et écrire}

\section{Renald Bérubé et Paul Chanel Malenfant}

Numéro 10, 2e trimestre 1984

Spécial fantasmes

URI : https://id.erudit.org/iderudit/025150ar

DOI : https://doi.org/10.7202/025150ar

Aller au sommaire du numéro

Éditeur(s)

Urgences

ISSN

0226-9554 (imprimé)

1927-3924 (numérique)

Découvrir la revue

Citer cet article

Bérubé, R. \& Malenfant, P. C. (1984). Texte à deux voix : l'enseignement de la littérature, lire et écrire. Urgences, (10), 7-15. https://doi.org/10.7202/025150ar d'utilisation que vous pouvez consulter en ligne.

https://apropos.erudit.org/fr/usagers/politique-dutilisation/ 


\title{
CHRONIQUE
}

TEXTE À DEUX VOIX: L'ENSEIGNEMENT DE LA LITTÉRATURE, LIRE ET ÉCRIRE

\author{
RENALD BÉRUBÉ \\ PAUL-CHANEL MALENFANT
}




\section{TEXTE À DEUX VOIX:}

\section{L'ENSEIGNEMENT DE LA LITTÉRATURE, LIRE ET ÉCRIRE}

\section{Variations sur la littérature et l'enseignement de la littérature à I'Université}

Et tout le reste est littérature, selon l'expression bien connue et souvent utilisée. Ce qui, on en conviendra aisément, laisse à la littérature un assez vaste champ - dont les contours restent souvent difficiles à distinguer, comme vagues parfois, mal balisés, et par là même mystérieux, attirants. Ou encore, pour prendre à rebours le raisonnement qui précède: balisé et surbalisé, le champ de la littérature n'en finit pas de se jouer des grilles et des clôtures, s'élargissant là où on croyait le contenir, ou se disséminant là, rusé, alors qu'on se croyait en tout autre terrain. Et si le "tout le reste est littérature", prononcé sur tel ton, laisse parfois (adverbe trop faible?), ironique, percer des connotations péjoratives, la littérature étant alors repoussée du côté de l'irréel, science (?) impure et inexacte, cela est peut-être lié au fait que la littérature, utilisant pourtant un instrument précis qui est aussi son objet, le langage, ne travaille pas que sur le rationnel et le quantifiable. Disant la vie à sa façon et la vie se manifestant de toutes les façons que I'on sait (et ne sait pas), la littérature ne peut que prendre toutes les formes en exploitant toutes les ressources du langage, repoussant même souvent les limites jusque-là admises de celui-ci. Et je pense à Borgès au sujet duquel Gérard Genette écrit: "... le mythe, fondamental chez lui, du Monde comme Bibliothèque (et Labyrinthe), qui ne lui donne accès aux choses et aux êtres qu'à travers les livres (1)"; de fait, toute bibliothèque contient (aussi) le labyrinthe du monde et la lecture se révèle bien une façon (parmi d'autres) d'accéder aux êtres et aux choses.

Enseigner la littérature alors, qu'est-ce à dire? Bien humblement, bien simplement, apprendre ( $s$ 'apprendre) à lire. Dans le sens où Goethe pouvait affirmer: "Les braves gens ne savent pas ce qu'il en coûte de temps et de peine pour apprendre à lire. J'ai travaillé à cela quatre-vingts ans, et je ne peux pas encore dire que j'y sois arrivé (2)"; dans le sens aussi de cette constatation un peu hésitante de Borges: "Je pense 
parfois que les bons lecteurs sont des oiseaux rares, encore plus ténébreux et singuliers que les bons auteurs... (3)". Enseignant ou étudiant la littérature, nous travaillons, "braves gens", à devenir (à former, si cela ne paraît pas trop prétentieux) des "oiseaux rares": de "bons lecteurs".

Ensemble, système de signes, I'oeuvre littéraire existe donc pour être lue, décodée aussi bien que faire se peut. Lecteur dont c'est le métier, je ferai appel, pour comprendre (lire) le mieux, le plus totalement possible, l'oeuvre que j'ai devant moi, à divers instruments, à diverses méthodes d'approche: histoire, sociologie, psychanalyse, linguistique, sémiologie, narratologie, etc. Le littéraire prend son bien où il le trouve, selon I'aspect de l'oeuvre qu'il veut étudier davantage ou encore selon la direction de lecture que commande l'oeuvre pour être mieux comprise. Ainsi, I'oeuvre sera replacée dans le contexte qui l'a vue naître, tel personnage sera analysé dans ses rapports à son milieu et à son entourage; mais surtout, la structure de l'oeuvre et son langage, les rapports que les divers éléments (mots, figures, thèmes, etc.) entretiennent et tissent entre eux, retiendront I'attention. Car si "Je suis triste et il pleut" (ou vice versa) d'une part, et "II pleure dans mon coeur/comme il pleut sur la ville" d'autre part, transmettent à la rigueur la même information, ils ne la formulent pas de la même façon et ne disent donc pas la même chose: la signification loge dans la formulation. De même, I'analyse menée par Serge Doubrovsky (4) permet de comprendre que le vers suivant du Cid de Corneille:

Don Diègue, par son fils, a vengé son offense ne dit pas seulement que Rodrigue a tué Don Gomès. II révèle aussi une conception du temps et de la personne, une organisation sociale, une façon d'être au monde.

Lire donc, apprendre (enseigner) à lire. Apprendre (enseigner) le sens multiple des mots, des signes, comme celui de leur choix et de leur agencement, et comprendre ainsi comment un univers se construit, est construit, nous parle, nous influence. La littérature mène à tout pourvu qu'on en sorte dit-on parfois (souvent?); je dirais plutôt qu'elle mène à tout pourvu qu'on n'en sorte pas. Car ne sommes-nous pas, constamment et quotidiennement, confronté à un travail de lecture? Dans un monde où l'image est reine quasi absolue, 
dans un monde où les idoles-stars-mythes-slogans (mot d'origine guerrière) se font et se défont au rythme de l'instantané (ou presque) et du prêt-à-penser, dans un monde où règnent les télécommunications, l'informatique, la bureaucratique et autres tictics d'ordinateur, il importe de savoir (bien) lire, décoder, interpréter, de posséder de solides outils de réflexion et d'analyse - sinon les images risquent de nous mener là où veulent nous diriger leurs concepteurs, à notre insu souvent, et à leur rythme. Selon la formule consacrée, même que le quart-arrière d'une équipe de football doit savoir "lire" le déploiement de la défensive adverse; il dispose pour ce faire de quelques rares secondes - et s'il se trompe, des arguments de poids et de taille le ramèneront vite à son livre de jeux, devant le tableau noir (vert?) de l'entraîneur. Bon (ah! les analogies...).

Apprendre à lire, c'est donc apprendre à parcourir le champ littéraire, à parcourir "le vaste monde" pour reprendre I'expression du Survenant. Reflet/transposition du monde, la littérature est aussi et en elle-même un univers: les oeuvres engendrent les oeuvres, lecture et création se donnant mutuellement naissance. "Chacun est fils de ses oeuvres", disait Don Quichotte qui est bien le fils de ses lectures - nous sommes aussi, tous, fils et filles de nos lectures. Ainsi, depuis Homère jusqu'à Joyce, Ulysse, à travers mutations, adaptations et transformations, demeure bien vivant; grâce à lui et en mettant en rapport les deux oeuvres, j'apprends ce qui dure et ce qui change, j'entends parler de la Grèce ancienne et de I'Irlande du début de ce siècle (et même, par la bande dessinée télévisée dont Ulysse est le héros, des âges à venir). Et à quoi ressemble Hamlet en Prince du Québec? Et pourquoi telle pratique littéraire, disons la mise en abyme, semble-t-elle davantage privilégiée à telle puis telle époque? Etudier cette pratique et les époques qui la privilégient, c'est tenter de comprendre ce qui, par-delà les siècles, peut relier deux âges (apparemment) bien éloignés. Et l'oeuvre de Faulkner, qui se déroule presque tout entière dans un coin bien circonscrit du Mississippi, n'en demeure pas moins de portée universelle: le monde se trouve dans cette oeuvre, dans ce coin de terre. Ce qui, vu (lu) du lieu que nous habitons, ne va pas sans enseignements précieux. 
Enseigner la littérature, apprendre à lire: apprendre à lire le monde et à me lire, à lire le monde en moi et à me lire dans le monde. Tout le reste est littérature - Hamlet disait que tout le reste est silence. Peut-être est-ce là, en fin de compte, la meilleure définition de la littérature: le silence qui s'écrit, se lit, s'écrit... Les mots du silencieux.

Son texte terminé, il le relut; la littérature, se dit-il alors, est affaire de musique: je joue des mots quand j'écris, j'interprète ceux des autres quand je lis. Voilà.

Renald Bérubé

UQAR

1. Gérard Genette, Palimpsestes, Paris, Seuil, "Poétique", 1982, p. 297.

2. Cité par Karl Petit, le Dictionnaire des citations du monde entier, Verviers, Marabout, 1960, p. 240.

3. Cité par Gérard Genette, Palimpsestes, p. 295-296.

4. Serge Doubrovsky, Corneille et la dialectique du héros, Paris, Gallimard, "Bibliothèque des idées", 1963, p. 87-132. 


\section{Lire: la plume à la main}

"Parler du langage est nécessairement passionnel" écrit Henri Meschonnic. De la part de l'érudit poéticien, cet aveu de passion (les séquelles d'une éducation sentimentale?...) m'interpelle, $m$ 'incline à une prudence logique, "syllogistique". Car si primo est: ..., secundo pourrait bien être, "L'enseignement de la littérature est nécessairement un langage" et, tertio: "L'enseignement de la littérature est nécessairement passionnel". Voilà posé un constat troublant, insidieux. Est-ce donc un métier de parler? (...) d'exhiber publiquement une passion pour le texte (sublimation latérale diront ceux qui ont lu Jung)? bref de proférer en un spectacle parlé la "mimesis interprétative" de la parole des écrivains (vol par effraction du sens accuseront les linguistes légistes)? Certes, ces questions sont piégées. Issue stratégique? Affirmer: au même titre que tous les hommes de sciences, le professeur de littérature est un savant. L'horlogerie du texte littéraire, il l'a mise à jour; n'a-t-il pas démontré la "littérarité" de Madame Bovary, expliqué le taux de "poéticité" du "sonnet en yx", défini les mécanismes pulsionnels et les structures internes de l'oeuvre? A preuve, lisons au hasard: Structures anthropologiques de l'imaginaire, Structures de la poésie moderne, Structures du langage poétique, La Structure absente... En outre, ce savant féru de théorie et de méthode (tel traité de narratologie ou de sémiotique: I'obliguité, I'intertextualité, l'agrammaticalisme...) a su, pa un légendaire labeur encyclopédique, s'inspirer des "autres" sciences. Tel pratique la sociocritique ou la psychocritque ou la mythocritique, tel autre se réfère à I'anthropologie ou à la phénoménologie à moins qu'il ne s'intéresse à la théorie des catastrophes, à l'herméneutique cybernétique ou aux racines biologiques du symbolique!... Ce savant-là "Ne (le) dérangez pas (il est) profondément occupé" (comme dans "Le jeu" de Saint-Denys Garneau). II compte des occurences, dissèque des syntagmes et des isotopes, il se ravit d'une analepse, hésite sur un trope, bref il s'adonne à la tâche, objectivement, scientifiquement, de démontrer les rouages de la vaste panoplie des chefs-d'oeuvre littéraires pour les rendre à des esprits avides de mécanique et de raison.

Au hasard de ses lectures, justement, ce docte professeur 
de littérature trébuche sur l'assertion péremptoire d'un de ses collègues d'outre-frontière, critique et professeur imminent, Serge Doubrovsky, "La vérité - gênante pour le professeur de littérature - est que la littérature ne s'enseigne pas". Et l'éminence (serait-elle grise?) surenchérit:

Quelle que soit l'étendue de son savoir, un professeur de littérature n'est pas un savant: son enseignement n'est pas une somme de renseignements; les vérités qu'il transmet, il les "véhicule", elles ne sont saisissables que "sur" lui; elles ne tiennent pas aux résultats qu'il communique, mais à "I'acte même" de la communication'.

Esprit contradicteur? La science de la littérature ne serait donc qu'un savoir sans connaissance, un contenu sans contenant? Le professeur ferait office de porte-parole de l'oeuvre, d'entremetteur, de second rôle ou d'actant? Que le langage - et tant va celui des professeurs - est source de malentendus! Volte-face. Antithèse. Stratégie du doute. Habitué de questionner voire de résoudre les oeuvres, le professeur hésite - mis en abyme - s'il doit interroger son propre texte interposé, celui qu'il porte sur les textes? (II lui faudrait ici les ressources de Miron ironisant: "J'ai une réponse. Vous avez une question?") Ainsi, qu'advient-il donc en cet acte de l'enseignement, qui est un discours, un discours au second degré? D'abord jugée suspecte, la passion refait surface et se donne pour science. Alors, tout se passe comme si l'enthousiasme solitaire de la lecture silencieuse (amoureuse) induisait une pulsion (une passion) de la parole publique, conduisait à une mise en scène de l'écriture lue. L'enseignement de la littérature relève de la lecture collective - la classe comme bibliothèque parlante - et de l'échange solidaire du sens multiple des oeuvres. Lire stimule un tel plaisir (ce "plaisir du texte", justement) qu'il engage la communication partagée de la jouissance. Erotisation textuelle, euphorie ou utopie? On s'étonne moins de cette exaltation si l'on considère que de plus en plus de professeurs de lettres (ces étudiants perpétuels) et d'étudiants (ces professeurs virtuels) écrivent. La lecture convoque non seulement la prise de la parole mais l'emprise de la plume, tant il est vrai 
qu'enseigner (qu'étudier) la littérature c'est, en un sens, la faire.

II faut se rendre à une certaine évidence: la littérature québécoise, de plus en plus et non la moindre (qu'on songe à Bessette, Noël, Villemaire, Théoret et les autres), est faite par des professeurs. La distance qu'on avait coutume d'établir entre le commentaire et la fiction tend à s'amenuiser dès que l'on accepte la part de fabulation dans l'un et de théorique dans l'autre, dès que l'on se rend compte que lire et écrire ne procèdent pas d'activités si distinctes, mais délicieusement jumelles et complémentaires. (Lise Gauvin, Le Devoir, samedi 25 février 1984, p. 19).

On peut entendre comme un signe notoire de santé pour une littérature, cette réversibilité, cette osmose empathique de la lecture à l'enseignement, de la lecture à l'écriture, de l'enseignement à l'écriture. En témoigne, il me semble, l'engouement de plus en plus marqué pour les ateliers littéraires ou pour des études supérieures axées tant sur la création d'une oeuvre personnelle que sur la réflexion théorique qui la supporte. (Se profilent, ici, ces programmes d'études littéraires dispensés dans diverses universités et qui offrent la possibilité d'un mémoire en création où tour à tour la fiction, la poésie ou l'essai s'accordent à la critique et à la théorie).

Cette unification polyvalente de l'activité littéraire trouve sans doute aussi son sens, depuis la fructueuse atténuation des impérialismes et des polémiques théoriques et méthodologiques (Bachelard contre Lacan, Todorov contre fohen), dans les accointances délibérées qui régissent les genres et les pratiques textuelles. Et qui ne saurait se réjouir que le ténébreux Barthes ait écrit les Fragments d'un discours amoureux, que l'imprenable Kristeva vienne de s'alléger d'Histoires d'amour, que le macho Sollers ait placé à l'avant-scène romanesque ses confidentes et joyeusement scandaleuses Femmes, enfin, ici et entre autres, que Nicole Brossard ait donné, après L'amer, Picture Theory? Ces croisements souvent passionnés de la fiction à la théorie, de la poésie à la "poétique", de la critique à la création illustrent la perméabilité qui assortit de plus en plus les zones diverses de la littérature. Dès lors, que 
les écrivains enseignent, que les professeurs et les étudiants écrivent, que la lecture se donne comme support intertextuel à l'écriture (la portée, soudain, de telle citation...), cette versatilité des interventions souligne l'accord capital qui s'instaure entre les revendications de liberté de l'objet littéraire et le désir accru de créativité de ceux qui s'exercent à le concevoir comme à le pratiquer dans l'enseignement. Alors, à la polyvalence des textes s'adjoint celle des fonctions qui le supportent.

Un regard rétrospectif sur ces lignes $m^{\prime}$ indique comment le parcours est interrogatif, oscillatoire entre le confort de la science, convoité sur le mode parodique, I'aveu de la passion littéraire et la quête d'une liberté créatrice. Enseigner la littérature? Ligne de partage entre les signes, ligne du risque et de dérive entre raison et imagination? Dans ce domaine, sans doute, peu de vérités qui soient des certitudes absolues; c'est bien pourquoi, la voix du professeur reste provisoire, toujours à reprendre, intermédiaire entre le "dit" de l'oeuvre et "I'autrement dit" de son propre discours. "Test projectif", déclare Barthes à propos de la critique; test subjectif et itinéraire prospectif, dirions-nous de l'enseignement. Si devant un texte littéraire des lecteurs parviennent ensemble à une productivité positive, ou de dévoilement des sens ou d'écritures concertantes, s'ils parlent pour que l'oeuvre "parle enfin", ils auront aussi compris qu' "En cette matière, prouver c'est éprouver ${ }^{2}$ ?"

Paul Chanel Malenfant UQAR

1. Serge DOUBROVSKY dans L'enseignement de la littérature, Paris, Plon, 1971, (Ouvrage en collaboration, Centre Culturel de Cerisy-la-Salle, sous la direction de Serge Doubrovsky et Tzvetan Todorov.

2. Serge DOUBROVSKY, Ibid. 